\title{
Farsightedly stable networks
}

Citation for published version (APA):

Herings, P. J. J., Mauleon, A., \& Vannetelbosch, V. (2009). Farsightedly stable networks. Games and Economic Behavior, 67, 526-541. https://doi.org/10.1016/j.geb.2008.12.009

Document status and date:

Published: 01/01/2009

DOI:

10.1016/j.geb.2008.12.009

Document Version:

Publisher's PDF, also known as Version of record

Document license:

Taverne

Please check the document version of this publication:

- A submitted manuscript is the version of the article upon submission and before peer-review. There can be important differences between the submitted version and the official published version of record.

People interested in the research are advised to contact the author for the final version of the publication, or visit the DOI to the publisher's website.

- The final author version and the galley proof are versions of the publication after peer review.

- The final published version features the final layout of the paper including the volume, issue and page numbers.

Link to publication

\footnotetext{
General rights rights.

- You may freely distribute the URL identifying the publication in the public portal. please follow below link for the End User Agreement:

www.umlib.nl/taverne-license

Take down policy

If you believe that this document breaches copyright please contact us at:

repository@maastrichtuniversity.nl

providing details and we will investigate your claim.
}

Copyright and moral rights for the publications made accessible in the public portal are retained by the authors and/or other copyright owners and it is a condition of accessing publications that users recognise and abide by the legal requirements associated with these

- Users may download and print one copy of any publication from the public portal for the purpose of private study or research.

- You may not further distribute the material or use it for any profit-making activity or commercial gain

If the publication is distributed under the terms of Article $25 \mathrm{fa}$ of the Dutch Copyright Act, indicated by the "Taverne" license above, 


\title{
Farsightedly stable networks
}

\author{
P. Jean-Jacques Herings ${ }^{\mathrm{a}, *}$, Ana Mauleon ${ }^{\mathrm{b}, \mathrm{c}}$, Vincent Vannetelbosch ${ }^{\mathrm{c}}$ \\ a Department of Economics, Maastricht University, P.O. Box 616, 6200 MD, Maastricht, The Netherlands \\ ${ }^{\mathrm{b}}$ FNRS and CEREC, Facultés Universitaires Saint-Louis, Boulevard du Jardin Botanique 43, B-1000 Brussels, Belgium \\ c FNRS and CORE, Université Catholique de Louvain, 34 voie du Roman Pays, B-1348 Louvain-la-Neuve, Belgium
}

\section{A R T I C L E I N F O}

\section{Article history:}

Received 8 April 2008

Available online 22 January 2009

\section{JEL classification:}

A14

C70

D20

\section{Keywords:}

Networks

Farsighted players

Stability

Pairwise deviations

Efficiency

\begin{abstract}
A B S T R A C T
A set of networks $G$ is pairwise farsightedly stable (i) if all possible farsighted pairwise deviations from any network $g \in G$ to a network outside $G$ are deterred by the threat of ending worse off or equally well off, (ii) if there exists a farsighted improving path from any network outside the set leading to some network in the set, and (iii) if there is no proper subset of $G$ satisfying conditions (i) and (ii). A non-empty pairwise farsightedly stable set always exists. We provide a full characterization of unique pairwise farsightedly stable sets of networks. Contrary to other pairwise concepts, pairwise farsighted stability yields a Pareto dominant network, if it exists, as the unique outcome. Finally, we study the relationship between pairwise farsighted stability and other concepts such as the largest pairwise consistent set and the von Neumann-Morgenstern pairwise farsightedly stable set.
\end{abstract}

(c) 2009 Elsevier Inc. All rights reserved.

\section{Introduction}

The organization of individual agents into networks and groups or coalitions plays an important role in the determination of the outcome of many social and economic interactions. For instance, networks of personal contacts are important in obtaining information on goods and services, like product information or information about job opportunities. Many commodities are traded through networks of buyers and sellers. The partitioning of societies into groups is also important in many contexts, such as the provision of public goods and the formation of alliances, cartels, and federations.

A simple way to analyze the networks that one might expect to emerge in the long run is to examine the requirement that individuals do not benefit from altering the structure of the network. An example of such a condition is the pairwise stability notion defined by Jackson and Wolinsky (1996). ${ }^{1}$ Their approach is static and myopic. Individuals are not forwardlooking in the sense that they do not forecast how others might react to their actions. For instance, individuals might not add a link that appears valuable to them given the current network, as that might in turn lead to the formation of other links and ultimately lower the payoffs of the original individuals.

A dynamic (but still myopic) network formation process has been recently studied by Jackson and Watts (2002), who have proposed a dynamic process in which individuals form and sever links based on the improvement that the resulting

\footnotetext{
* Corresponding author.

E-mail addresses: P.Herings@algec.unimaas.nl (P.J.J. Herings), mauleon@fusl.ac.be (A. Mauleon), vincent.vannetelbosch@uclouvain.be (V. Vannetelbosch).

1 There are alternative ways to model network stability. One is to explicitly model a game by which links form and then to solve that game using the concept of Nash equilibrium or one of its refinements. See Aumann and Myerson (1988) and Dutta and Mutuswami (1997). Jackson (2003, 2005) provides surveys of models of network formation.
} 
network offers them relative to the current network. This deterministic dynamic process may end at a pairwise stable network or may cycle.

In this paper we address the question which networks one might expect to emerge in the long run when players are farsighted. Since most of the literature considers the case where at most one link is changed at a time, we will also restrict our analysis to network formation processes with this characteristic. This enables us to make the best comparison of our results to those found in the literature. It is straightforward to adapt our concept to more general network formation processes.

We first extend the Jackson and Wolinsky pairwise stability notion to a new set-valued solution concept, called the pairwise myopically stable set. A set of networks $G$ is pairwise myopically stable (i) if all possible myopic pairwise deviations from any network $g \in G$ to a network outside the set are deterred by the threat of ending worse off or equally well off, (ii) if there exists a myopic improving path from any network outside the set leading to some network in the set, and (iii) if there is no proper subset of $G$ satisfying conditions (i) and (ii). We show that there is a unique pairwise myopically stable set and that it is equal to the collection of closed cycles. It follows that the pairwise myopically stable set is non-empty and contains all pairwise stable networks.

We then introduce the pairwise farsightedly stable set, to predict which networks may be formed among farsighted players. The definition corresponds to the one of a pairwise myopically stable set with myopic deviations and myopic improving paths replaced by farsighted deviations and farsighted improving paths. A farsighted improving path is a sequence of networks that can emerge when players form or sever links based on the improvement the end network offers relative to the current network. Each network in the sequence differs by one link from the previous one. If a link is added, then the two players involved must both prefer the end network to the current network, with at least one of the two strictly preferring the end network. If a link is deleted, then it must be that at least one of the two players involved in the link strictly prefers the end network.

In contrast to other concepts incorporating farsightedness, we do not only request that all possible pairwise deviations out of the set are deterred by the threat of ending worse off, but also that there exists a farsighted improving path from any network outside the set leading to some network in the set. This property is equivalent to the requirement that networks within the set are robust to perturbations. Perturbations may be due to exogenous forces acting on the network, or simply miscalculations or errors on the part of an individual making an assessment or taking an action. ${ }^{2}$

We show that a pairwise farsightedly stable set always exists and we provide a full characterization of unique pairwise farsightedly stable sets of networks. As a corollary, we give the necessary and sufficient condition such that a unique pairwise farsightedly stable set consisting of a single network exists. We apply these results to examples, such as the criminal network model of Calvó-Armengol and Zenou (2004). We find that in criminal networks with $n$ players, the set consisting of the complete network (where all criminals are linked to each other) is a pairwise farsightedly stable set.

We consider the relationship between farsighted stability and efficiency of networks. We provide conditions under which pairwise farsighted stability singles out a strongly efficient network. We show that if there is a network that Pareto dominates all other networks, then that network is the unique prediction of pairwise farsighted stability. This property does not hold for other pairwise solution concepts.

Finally, we study the relationship between pairwise farsighted stability and other farsighted concepts such as the largest pairwise consistent set, a notion due to Chwe (1994), and the von Neumann-Morgenstern pairwise farsightedly stable set. Under some conditions, a pairwise farsightedly stable set is a subset of the set of pairwise stable networks, which in turn is a subset of the largest pairwise consistent set. We show that any von Neumann-Morgenstern pairwise farsightedly stable set is also a pairwise farsightedly stable set. Under some conditions, also the reverse statement holds. By means of examples we show that pairwise farsightedly stable sets have no relationship to either largest pairwise consistent sets or pairwise myopically stable sets.

Although the literature on stability in networks is well established and growing (see Jackson, 2005), the literature on farsighted stability is still in its infancy. Page Jr. et al. (2005) address the issue of farsighted stability in network formation by extending Chwe's (1994) result on the nonemptiness of farsightedly consistent sets. In order to demonstrate the existence of farsightedly consistent directed networks, they provide a new framework that extends the standard notion of a directed network and also introduces the notion of a supernetwork. A supernetwork specifies how the different directed networks are connected via coalitional moves and coalitional preferences, and thus provides a network representation of agent preferences and the rules governing network formation. A supernetwork is equivalent to the social environment studied by Chwe (1994), when the set of outcomes is replaced by the set of directed networks. Given the rules governing network formation and agents' preferences as represented via the supernetwork, a directed network (i.e., a particular node in the supernetwork) is said to be farsightedly consistent if no agent or coalition of agents is willing to alter the network (via the addition, subtraction, or replacement of arcs) in fear that such an alteration might induce further network alterations by other agents or coalitions that in the end leave the initially deviating agent or coalition no better off, and possibly worse off. They have shown that for any supernetwork corresponding to a given collection of directed networks, the set of farsightedly consistent networks is non-empty; see also Page Jr. et al. (2005).

\footnotetext{
${ }^{2}$ Jackson and Watts (2002) use improving paths as the foundation for a stochastic analysis, where in addition to intended changes in the network, unintended mutations or errors are introduced. However, in their definition of improving path it is assumed that players behave myopically: all a player needs to know is whether adding or deleting a given link is directly beneficial to him or her under the current circumstances.
} 
Dutta et al. (2005) have studied a model of dynamic network formation where individuals are farsighted and evaluate the desirability of a move in terms of its consequences on the entire discounted stream of payoffs. Contrary to ours, their model is in spirit closer to non-cooperative game theory. They show that a Markovian equilibrium process of network formation exists and they provide two conditions, link monotonicity and increasing returns to link creation, each of which guarantees that there is some equilibrium at which the complete graph is reached in the limit from all initial networks. They also show that there are valuation structures in which the process will not converge to any efficient network for any equilibrium strategy profile. This can be viewed as the dynamic counterpart of the conflict between static stability and efficiency demonstrated by Jackson and Wolinsky (1996), a conflict that is also confirmed by our results. Dutta et al. (2005) provide an example where there is a network that Pareto dominates all other networks, but which is not reached in equilibrium. In our framework, such an example is not possible. If there is a network that Pareto dominates all other networks, then that network is the unique prediction of pairwise farsighted stability. Other approaches to farsightedness in network formation are suggested by the work of Xue (1998), Herings et al. (2004), and Mauleon and Vannetelbosch (2004).

The paper is organized as follows. In Section 2 we introduce some notations and basic properties and definitions for networks. In Section 3 we define the notion of pairwise myopically stable set of networks. In Section 4 we define the notion of pairwise farsightedly stable set of networks and we characterize it in Section 5. In Section 6 we consider the symmetric connections model and the co-author model. We look at the relationship between farsighted stability and efficiency of networks in Section 7. In Sections 8 and 9 we analyze, respectively, the relationship with the von Neumann-Morgenstern pairwise farsightedly stable set and the largest pairwise consistent set. Finally, in Section 10 we conclude.

\section{Networks}

Let $N=\{1, \ldots, n\}$ be the finite set of players who are connected in some network relationship. The network relationships are reciprocal and the network is thus modeled as a non-directed graph. Individuals are the nodes in the graph and links indicate bilateral relationships between individuals. Thus, a network $g$ is simply a list of which pairs of individuals are linked to each other. We write $i j \in g$ to indicate that $i$ and $j$ are linked under the network $g$. Let $g^{N}$ be the collection of all subsets of $N$ with cardinality 2 , so $g^{N}$ is the complete network. The set of all possible networks or graphs on $N$ is denoted by $\mathbb{G}$ and consists of all subsets of $g^{N}$. The network obtained by adding link $i j$ to an existing network $g$ is denoted $g+i j$ and the network that results from deleting link $i j$ from an existing network $g$ is denoted $g-i j$. For any network $g$, let $N(g)=\{i \mid$ there is $j$ such that $i j \in g\}$ be the set of players who have at least one link in the network $g$. A path in a network $g \in \mathbb{G}$ between $i$ and $j$ is a sequence of players $i_{1}, \ldots, i_{K}$ such that $i_{k} i_{k+1} \in g$ for each $k \in\{1, \ldots, K-1\}$ with $i_{1}=1$ and $i_{K}=j$. A non-empty network $h \subseteq g$ is a component of $g$, if for all $i \in N(h)$ and $j \in N(h) \backslash\{i\}$, there exists a path in $h$ connecting $i$ and $j$, and for any $i \in N(h)$ and $j \in N(g), i j \in g$ implies $i j \in h$. The set of components of $g$ is denoted by $C$ ( $g$ ). Knowing the components of a network, we can partition the players into groups within which players are connected. Let $\Pi(\mathrm{g})$ denote the partition of $N$ induced by the network $g .^{3}$

A value function is a function $v: \mathbb{G} \rightarrow \mathbb{R}$ that keeps track of how the total societal value varies across different networks. The set of all possible value functions is denoted by $\mathcal{V}$. An allocation rule is a function $Y: \mathbb{G} \times \mathcal{V} \rightarrow \mathbb{R}^{N}$ that keeps track of how the value is allocated or distributed among the players forming a network. It satisfies $\sum_{i \in N} Y_{i}(g, v)=v(g)$ for all $v$ and $g$.

Jackson and Wolinsky (1996) have proposed a number of basic properties of value and allocation functions. A value function is component additive if $v(g)=\sum_{h \in C(g)} v(h)$ for all $g \in \mathbb{G}$. Component additive value functions are the ones for which the value of a network is the sum of the value of its components. An allocation rule $Y$ is component balanced if for any component additive $v \in \mathcal{V}, g \in \mathbb{G}$, and $h \in C(g)$, we have $\sum_{i \in N(h)} Y_{i}(h, v)=v(h)$. Component balancedness only puts conditions on $Y$ for $v$ 's that are component additive, so $Y$ can be arbitrary otherwise. Given a permutation of players $\pi$ and any $g \in \mathbb{G}$, let $g^{\pi}=\{\pi(i) \pi(j) \mid i j \in g\}$. Thus, $g^{\pi}$ is a network that is identical to $g$ up to a permutation of the players. A value function is anonymous if for any permutation $\pi$ and any $g \in \mathbb{G}, v\left(g^{\pi}\right)=v(g)$. Given a permutation $\pi$, let $v^{\pi}$ be defined by $v^{\pi}(g)=v\left(g^{\pi^{-1}}\right)$ for each $g \in \mathbb{G}$. An allocation rule $Y$ is anonymous if for any $v \in \mathcal{V}, g \in \mathbb{G}$, and permutation $\pi$, we have $Y_{\pi(i)}\left(g^{\pi}, v^{\pi}\right)=Y_{i}(g, v) .^{4}$

An allocation rule that is component balanced and anonymous is the componentwise egalitarian allocation rule. For a component additive $v$ and network $g$, the componentwise egalitarian allocation rule $Y^{c e}$ is such that for any $h \in C(g)$ and each $i \in N(h), Y_{i}^{c e}(g, v)=v(h) / \# N(h)$. For a $v$ that is not component additive, $Y^{c e}(g, v)=v(g) / n$ for all $g$; thus, $Y^{c e}$ splits the value $v(g)$ equally among all players if $v$ is not component additive.

In evaluating societal welfare, we may take various perspectives. A network $g$ is Pareto efficient relative to $v$ and $Y$ if there does not exist any $g^{\prime} \in \mathbb{G}$ such that $Y_{i}\left(g^{\prime}, v\right) \geqslant Y_{i}(g, v)$ for all $i$ with at least one strict inequality. A network $g \in \mathbb{G}$ is strongly efficient relative to $v$ if $v(g) \geqslant v\left(g^{\prime}\right)$ for all $g^{\prime} \in \mathbb{G}$. This is a strong notion of efficiency as it takes the perspective that value is fully transferable.

\footnotetext{
3 Throughout the paper we use the notation $\subseteq$ for weak inclusion and $\varsubsetneqq$ for strict inclusion. Finally, \# will refer to the notion of cardinality.

4 Anonymous value functions are those such that the architecture of a network matters, but not the labels of individuals. Anonymity of an allocation rule requires that if only the labels of the agents change and the value generated by networks changes in an exactly corresponding fashion, then the allocation only changes according to the relabeling.
} 
The network-theoretic literature uses two different notions of deviation by a coalition. Pairwise deviations (Jackson and Wolinsky, 1996) are deviations involving a single link at a time. Moreover, link addition is bilateral (two players that would be involved in the link must agree to adding the link), link deletion is unilateral (at least one player involved in the link must agree to deleting the link), and network changes take place one link at a time. Coalitionwise deviations (Jackson and van den Nouweland, 2005) are deviations involving several links and some group of players at a time. Link addition is bilateral, link deletion is unilateral, and multiple link changes can take place at a time. Whether a pairwise deviation or a coalitionwise deviation makes more sense will depend on the setting within which network formation takes place.

We will restrict our analysis to pairwise deviations. A simple way to analyze the networks that one might expect to emerge in the long run is to examine the requirement that agents do not benefit from altering the structure of the network. A weak version of such a condition is the pairwise stability notion defined by Jackson and Wolinsky (1996). A network is pairwise stable if no player benefits from severing one of their links and no other two players benefit from adding a link between them, with one benefiting strictly and the other at least weakly. Formally, a network $g$ is pairwise stable with respect to value function $v$ and allocation rule $Y$ if

(i) for all $i j \in g, Y_{i}(g, v) \geqslant Y_{i}(g-i j, v)$ and $Y_{j}(g, v) \geqslant Y_{j}(g-i j, v)$, and

(ii) for all $i j \notin g$, if $Y_{i}(g, v)<Y_{i}(g+i j, v)$ then $Y_{j}(g, v)>Y_{j}(g+i j, v)$.

We say that $g^{\prime}$ is adjacent to $g$ if $g^{\prime}=g+i j$ or $g^{\prime}=g-i j$ for some $i j$. A network $g^{\prime}$ defeats $g$ if either $g^{\prime}=g-i j$ and $Y_{i}\left(g^{\prime}, v\right)>Y_{i}(g, v)$ or $Y_{j}\left(g^{\prime}, v\right)>Y_{j}(g, v)$, or if $g^{\prime}=g+i j$ with $Y_{i}\left(g^{\prime}, v\right) \geqslant Y_{i}(g, v)$ and $Y_{j}\left(g^{\prime}, v\right) \geqslant Y_{j}(g, v)$ with at least one inequality holding strictly. Pairwise stability is equivalent to the statement of not being defeated by another network..$^{5}$

\section{Pairwise myopically stable sets of networks}

Pairwise stable networks do not always exist. Following Jackson and Watts (2002), we introduce the notion of myopic improving path. A myopic improving path is a sequence of networks that can emerge when players form or sever links based on the improvement the resulting network offers relative to the current network. Each network in the sequence differs by one link from the previous one. If a link is added, then the two players involved must both prefer the resulting network to the current network, with at least one of the two strictly preferring the resulting network. If a link is deleted, then it must be that at least one of the two players involved in the link strictly prefers the resulting network.

Definition 1. A myopic improving path from a network $g$ to a network $g^{\prime} \neq g$ is a finite sequence of graphs $g_{1}, \ldots, g_{K}$ with $g_{1}=g$ and $g_{K}=g^{\prime}$ such that for any $k \in\{1, \ldots, K-1\}$ either:

(i) $g_{k+1}=g_{k}-i j$ for some $i j$ such that $Y_{i}\left(g_{k+1}, v\right)>Y_{i}\left(g_{k}, v\right)$ or $Y_{j}\left(g_{k+1}, v\right)>Y_{j}\left(g_{k}, v\right)$, or

(ii) $g_{k+1}=g_{k}+i j$ for some $i j$ such that $Y_{i}\left(g_{k+1}, v\right)>Y_{i}\left(g_{k}, v\right)$ and $Y_{j}\left(g_{k+1}, v\right) \geqslant Y_{j}\left(g_{k}, v\right)$.

A myopic improving path is a sequence of adjacent networks that might be observed in a dynamic process where players are adding and deleting links, one at a time. If there exists a myopic improving path from $g$ to $g^{\prime}$, then we write $g \mapsto g^{\prime}$. For a given network $g$, let $M(g)=\left\{g^{\prime} \in \mathbb{G} \mid g \mapsto g^{\prime}\right\}$. This is the set of networks that can be reached by a myopic improving path from $g$. Thus, $g \mapsto g^{\prime}$ means that $g^{\prime}$ is the endpoint of at least one myopic improving path from $g$. Notice that if $g$ is pairwise stable then $M(g)=\emptyset$.

It is well known that there are many allocation rules and value functions for which pairwise stable networks do not exist. We therefore consider a set-valued solution concept that captures the pairwise stability notion, called the pairwise myopically stable set of networks.

Definition 2. A set of networks $G \subseteq \mathbb{G}$ is pairwise myopically stable with respect $v$ and $Y$ if

(i) $\forall g \in G$,

(ia) $\forall i j \notin g$ such that $g+i j \notin G,\left(Y_{i}(g+i j, v), Y_{j}(g+i j, v)\right)=\left(Y_{i}(g, v), Y_{j}(g, v)\right)$ or $Y_{i}(g+i j, v)<Y_{i}(g, v)$ or $Y_{j}(g+$ $i j, v)<Y_{j}(g, v)$,

(ib) $\forall i j \in g$ such that $g-i j \notin G, Y_{i}(g-i j, v) \leqslant Y_{i}(g, v)$ and $Y_{j}(g-i j, v) \leqslant Y_{j}(g, v)$,

(ii) $\forall g^{\prime} \in \mathbb{G} \backslash G, M\left(g^{\prime}\right) \cap G \neq \emptyset$,

(iii) $\nexists G^{\prime} \varsubsetneqq G$ such that $G^{\prime}$ satisfies conditions (ia), (ib), and (ii).

Conditions (ia) and (ib) in Definition 2 capture deterrence of external deviations. In condition (ia) the addition of a link $i j$ to a network $g \in G$ that leads to a network outside $G$ is deterred because the two players involved do not prefer the

\footnotetext{
${ }^{5}$ Jackson and van den Nouweland (2005) have proposed a refinement of pairwise stability where coalitionwise deviations are allowed: the strongly stable networks. A strongly stable network is a network which is stable against changes in links by any coalition of individuals. Strongly stable networks are Pareto efficient and maximize the overall value of the network if the value of each component of a network is allocated equally among the members of that component.
} 
resulting network to network $g$. Condition (ib) is a similar requirement, but then for the case where a link is severed. Condition (ii) requires external stability. External stability asks for the existence of a myopic improving path from any network outside $G$ leading to some network in $G$. Condition (ii) implies that if a set of networks is pairwise myopically stable, it is non-empty. Notice that the set $\mathbb{G}$ (trivially) satisfies conditions (ia), (ib), and (ii) in Definition 2 . This motivates condition (iii), the minimality condition.

Jackson and Watts (2002) define the notion of a closed cycle. A set of networks $C$ is a cycle if for any $g \in C$ and $g^{\prime} \in C \backslash\{g\}$, there exists a myopic improving path connecting $g$ to $g^{\prime}$. A cycle $C$ is a maximal cycle if it is not a proper subset of a cycle. A cycle $C$ is a closed cycle if no network in $C$ lies on a myopic improving path leading to a network that is not in $C$. A closed cycle is necessarily a maximal cycle. If $g$ is a pairwise stable network, then trivially $\{g\}$ is a closed cycle.

Lemma 1. For every $g \in \mathbb{G}$, either $g$ is pairwise stable or there is a closed cycle $C$ such that $C \subseteq M(g)$.

Proof. Consider a network $g \in \mathbb{G}$ that is not pairwise stable. Construct a sequence of networks $g_{1}, \ldots, g_{k}$ such that $g_{1}=g$, $g_{j+1} \in M\left(g_{j}\right)$ for $j=1, \ldots, k-1$, and either $M\left(g_{k}\right)=\emptyset$ or $g_{k}=g_{j}$ for some $j<k$. In the former case, $g_{k}$ is pairwise stable and by transitivity of $M,\left\{g_{k}\right\} \subseteq M(g)$. In the latter case, let $C^{1}$ be a maximal cycle containing $\left\{g_{j}, \ldots, g_{k-1}\right\}$. Either $C^{1}$ is a closed cycle and we are done, or it has a myopic improving path going out of it, leading to a new maximal cycle $C^{2}$. Repeating this argument we reach after a finite number of steps a maximal cycle without myopic improving paths going out of it, i.e. a closed cycle.

Lemma 1 confirms the result of Jackson and Watts (2002) that for any value function and allocation rule there exists at least one closed cycle of networks. The next result claims that there is a unique pairwise myopically stable set of networks. It contains all networks that belong to a closed cycle.

Theorem 1. The set of networks consisting of all networks that belong to a closed cycle is the unique pairwise myopically stable set.

Proof. Let $G$ be the set consisting of all networks that belong to a closed cycle. We show that $G$ satisfies conditions (i)-(iii). Obviously, $G$ satisfies condition (i). The set $G$ also satisfies condition (ii). Indeed, consider some $g^{\prime} \notin G$. By Lemma 1 , either $g^{\prime}$ is pairwise stable or there is a closed cycle $C$ such that $C \subseteq M\left(g^{\prime}\right)$. The former case contradicts $g^{\prime} \notin G$. The latter case implies $g \in M\left(g^{\prime}\right)$ for some $g \in G$, i.e. implies condition (ii). Suppose $G$ does not satisfy condition (iii). Let $G^{\prime} \varsubsetneqq G$ satisfy conditions (i) and (ii). Let $g$ be a network that belongs to $G$ but not to $G^{\prime}$. If $g$ is pairwise stable, then there is no $g^{\prime} \in G^{\prime}$ such that $g^{\prime} \in M(g)$, so $G^{\prime}$ violates condition (ii). The network $g$ is therefore part of a closed cycle $C$ consisting of at least two networks. Moreover, for every $\bar{g} \in C$ it holds that $g \in M(\bar{g})$. If $C \cap G^{\prime}=\emptyset$, we violate condition (ii). If $C \cap G^{\prime}$ contains a network $\bar{g}$, then $g \in M(\bar{g})$ implies that condition (i) is violated. It follows that a set $G^{\prime} \varsubsetneqq G$ satisfying conditions (i) and (ii) does not exist, so $G$ is a pairwise myopically stable set.

Next we show that if a set of networks $G$ satisfies conditions (i)-(iii), then $G$ consists of all networks that belong to a closed cycle. If $G$ does not contain a pairwise stable network, then we have a contradiction to condition (ii). If $G$ does not contain some network that belongs to a closed cycle with at least two elements, then we have a contradiction to condition (i). It follows that $G$ contains all networks that belong to a closed cycle. Condition (iii) together with the first part of the proof now yields that $G$ cannot contain any other networks.

Sengupta and Sengupta (1994) define an indirect dominance relation for transferable utility games in coalition structure that is analogous to our notion of an improving path. Following them, we can define a network $g$ to be viable if for every network $g^{\prime} \in M(g)$ it holds that $g \in M\left(g^{\prime}\right)$. It is easily verified that any viable network $g$ belongs to a closed cycle. Indeed, if $g$ is viable, then $M(g)$ is a closed cycle, and vice versa. We can therefore rephrase Theorem 1 as the statement that the unique pairwise myopically stable set coincides with the set of viable networks.

\section{Pairwise farsightedly stable sets of networks}

We start this section with an example that shows the limitations of the pairwise myopically stable set and that motivates the incorporation of an appropriate notion of farsightedness.

Example 1 (Criminal networks). ${ }^{6}$ Each player is a criminal. If two players are connected, then they are part of the same criminal network. Each group of connected criminals has a positive probability of winning the loot. The loot is divided among the connected criminals based on the network architecture. Criminal $i$ 's payoff is given by

$$
\begin{aligned}
Y_{i}(g) & =p_{i}(g) \cdot\left[y_{i}(g) \cdot(1-\phi)\right]+\left(1-p_{i}(g)\right) \cdot y_{i}(g) \\
& =y_{i}(g) \cdot\left[1-p_{i}(g) \cdot \phi\right],
\end{aligned}
$$

\footnotetext{
6 This is a simplified version of Calvó-Armengol and Zenou (2004) where, in addition to forming links with criminal mates, criminals choose their level of criminal activities and whether or not to be involved in criminal activities.
} 


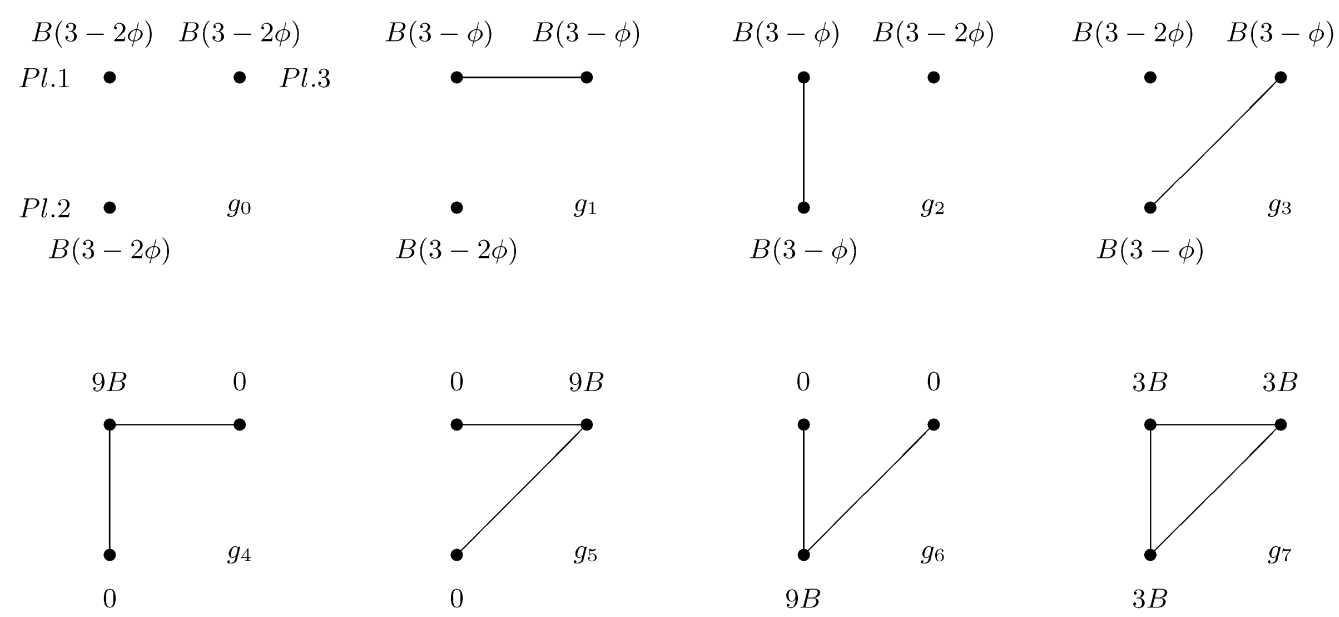

Fig. 1. Criminal networks.

where $y_{i}(g)$ is $i$ 's expected share of the loot, $p_{i}(g)$ is $i$ 's probability of being caught, and $\phi>0$ is the penalty rate. ${ }^{7}$ Beside being competitors in the crime market, criminals may also benefit from having criminal mates. It is assumed that (i) the bigger the group of connected criminals, the higher its probability of getting the loot, and (ii) the higher the number of links a criminal has, the lower his individual probability of being caught. Suppose the probability of being caught for criminal $i$ is simply given by

$$
p_{i}(g)=\frac{n-1-n_{i}}{n},
$$

with $n_{i}$ the number of links criminal $i$ has. ${ }^{8}$ For any group $S \in \Pi(g)$ of connected criminals, let $\bar{n}(S)=\max _{i \in S}\left[n_{i}\right]$. A criminal $i$ that is part of a group $S \in \Pi(g)$ expects a share of the loot $B>0$ given by

$$
y_{i}(g)=\frac{|S|}{n} \cdot \alpha_{i}(g) \cdot B
$$

where $|S| / n$ is the probability that group $S$ will win the loot, and $\alpha_{i}(g)$ is the share of the loot criminal $i \in S$ would obtain, which is given by

$$
\alpha_{i}(g)= \begin{cases}\frac{1}{\#\left\{j \in S \mid n_{j}=\bar{n}(S)\right\}} & \text { if } n_{i}=\bar{n}(S), \\ 0 & \text { otherwise. }\end{cases}
$$

That is, within each component, the criminal who has the highest number of links gets the loot. If two or more criminals have the highest number of links, then they share the loot equally among them. In Fig. 1 we have depicted the 3-player case with all payoffs in $1 / 9$ th's. For $\phi<3 / 2$, both the partial networks $\left(g_{1}, g_{2}, g_{3}\right)$ and the complete network $\left(g_{7}\right)$ are pairwise stable networks. There are four closed cycles, $\left\{g_{1}\right\},\left\{g_{2}\right\},\left\{g_{3}\right\}$, and $\left\{g_{7}\right\}$. The pairwise myopically stable set of networks is therefore given by $\left\{g_{1}, g_{2}, g_{3}, g_{7}\right\}$. For $\phi \geqslant 3 / 2$, the complete network $g_{7}$ is the only pairwise stable network. There is only one closed cycle, $\left\{g_{7}\right\}$, which is therefore also the pairwise myopically stable set of networks.

Take some strictly positive $\phi$ smaller than $3 / 2$ in Example 1 . The partial networks $g_{1}, g_{2}$, and $g_{3}$ are pairwise stable. Notice that two links have to be added to a partial network $g_{1}, g_{2}$, or $g_{3}$ to form the complete network $g_{7}$. Farsighted players may decide to add one link to a network like $g_{1}, g_{2}$, or $g_{3}$, accepting a loss, in the expectation that a further link will be added to form the complete network. A farsighted improving path is a sequence of networks that can emerge when players form or sever links based on the improvement the end network offers relative to the current network. Each network in the sequence differs by one link from the previous one. If a link is added, then the two players involved must both prefer the end network to the current network, with at least one of the two strictly preferring the end network. If a link is deleted, then it must be that at least one of the two players involved in the link strictly prefers the end network. We now introduce the formal definition of a farsighted improving path.

Definition 3. A farsighted improving path from a network $g$ to a network $g^{\prime} \neq g$ is a finite sequence of graphs $g_{1}, \ldots, g_{K}$ with $g_{1}=g$ and $g_{K}=g^{\prime}$ such that for any $k \in\{1, \ldots, K-1\}$ either:

\footnotetext{
7 The value function $v$ is simply $v(g)=\sum_{i \in N} Y_{i}(g)$. Since $v$ is fixed, we omit it in the notation of $Y_{i}(v, g)$.

8 This assumption captures the idea that delinquents learn from other criminals belonging to the same network how to commit crime in a more efficient way by sharing the know-how about the technology of crime (see Calvó-Armengol and Zenou, 2004).
} 
(i) $g_{k+1}=g_{k}-i j$ for some $i j$ such that $Y_{i}\left(g_{K}, v\right)>Y_{i}\left(g_{k}, v\right)$ or $Y_{j}\left(g_{K}, v\right)>Y_{j}\left(g_{k}, v\right)$, or

(ii) $g_{k+1}=g_{k}+i j$ for some $i j$ such that $Y_{i}\left(g_{K}, v\right)>Y_{i}\left(g_{k}, v\right)$ and $Y_{j}\left(g_{K}, v\right) \geqslant Y_{j}\left(g_{k}, v\right)$.

If there exists a farsighted improving path from $g$ to $g^{\prime}$, then we write $g \rightarrow g^{\prime}$. For a given network $g$, let $F(g)=\left\{g^{\prime} \in\right.$ $\left.\mathbb{G} \mid g \rightarrow g^{\prime}\right\}$. This is the set of networks that can be reached by a farsighted improving path from $g$. Thus, $g \rightarrow g^{\prime}$ means that $g^{\prime}$ is the endpoint of at least one farsighted improving path from $g$. Notice that $F(g)$ may contain many networks and that a network $g^{\prime} \in F(g)$ might be the endpoint of several farsighted improving paths starting in $g$. Since we are interested in stability of networks, there will be no need to specify on which particular path players eventually agree. Rather $F$ ( $g$ ) represents the networks that could possibly be reached by farsighted players when starting in $g$, and our concept of stability takes these possible end networks into account in a way that we will make precise in Definition 4.

The notion of farsightedness is relevant whenever payoffs can only be reaped after some stable network has formed, or when players are sufficiently patient so that they can safely ignore the payoffs that they obtain before a stable network settles down. It lies at the other end of the spectrum than myopia, where only immediate payoffs count. An intermediate (but difficult) approach is the one of Dutta et al. (2005), where the entire discounted stream of payoffs matters.

Suppose in Example 1 with $\phi$ smaller than $3 / 2$ that the starting network $g$ is a partial network like $g_{1}, g_{2}$, or $g_{3}$. Then, from $g$ no myopic improving path results in the complete network. The problem is that the isolated player will loose from making a link with any of the other players. However, there are farsighted improving paths that go to the complete network. An example of the sequence of graphs on a farsighted improving path is $\left(g_{1}, g_{4}, g_{7}\right)$ when starting in $g_{1}$. Similar farsighted improving paths exist starting in any of the other partial networks. Examples of other farsighted improving paths starting in $g_{1}$ and ending in $g_{7}$ are $\left(g_{1}, g_{4}, g_{7}\right),\left(g_{1}, g_{5}, g_{7}\right)$, or even $\left(g_{1}, g_{0}, g_{2}, g_{6}, g_{7}\right)$. Moreover, from any $g \neq g_{7}$ there is a farsighted improving path going to $g_{7}$. Thus, we observe that the partial networks are pairwise stable, but not stable when players are farsighted. The complete network on the other hand is not only pairwise stable. It is also stable when players are farsighted.

We now introduce a new solution concept, the pairwise farsightedly stable set. The definition corresponds to the one of a pairwise myopically stable set with myopic deviations replaced by farsighted deviations. It is obtained by requiring the deterrence of farsighted external deviations, farsighted external stability, and minimality. More precisely, a set of networks $G$ is pairwise farsightedly stable if (i) all possible pairwise deviations from any network $g \in G$ to a network outside $G$ are deterred by a credible threat of ending worse off or equally well off, (ii) there exists a farsighted improving path from any network outside the set leading to some network in the set, and (iii) there is no proper subset of $G$ satisfying conditions (i) and (ii). Formally, pairwise farsightedly stable sets are defined as follows.

Definition 4. A set of networks $G \subseteq \mathbb{G}$ is pairwise farsightedly stable with respect $v$ and $Y$ if

(i) $\forall g \in G$,

(ia) $\forall i j \notin g$ such that $g+i j \notin G, \exists g^{\prime} \in F(g+i j) \cap G$ such that $\left(Y_{i}\left(g^{\prime}, v\right), Y_{j}\left(g^{\prime}, v\right)\right)=\left(Y_{i}(g, v), Y_{j}(g, v)\right)$ or $Y_{i}\left(g^{\prime}, v\right)<$ $Y_{i}(g, v)$ or $Y_{j}\left(g^{\prime}, v\right)<Y_{j}(g, v)$,

(ib) $\forall i j \in g$ such that $g-i j \notin G, \exists g^{\prime}, g^{\prime \prime} \in F(g-i j) \cap G$ such that $Y_{i}\left(g^{\prime}, v\right) \leqslant Y_{i}(g, v)$ and $Y_{j}\left(g^{\prime \prime}, v\right) \leqslant Y_{j}(g, v)$,

(ii) $\forall g^{\prime} \in \mathbb{G} \backslash G, F\left(g^{\prime}\right) \cap G \neq \emptyset$.

(iii) $\nexists G^{\prime} \varsubsetneqq G$ such that $G^{\prime}$ satisfies conditions (ia), (ib), and (ii).

Condition (ia) in Definition 4 captures that adding a link $i j$ to a network $g \in G$ that leads to a network outside of $G$, is deterred by the threat of ending in $g^{\prime}$. Here $g^{\prime}$ is such that there is a farsighted improving path from $g+i j$ to $g^{\prime}$. Moreover, $g^{\prime}$ belongs to $G$, which makes $g^{\prime}$ a credible threat. Condition (ib) is a similar requirement, but then for the case where a link is severed. Condition (ii) in Definition 4 requires external stability and implies that the networks within the set are robust to perturbations. From any network outside $G$ there is a farsightedly stable path leading to some network in $G .{ }^{9}$ Condition (ii) implies that if a set of networks is pairwise farsightedly stable, it is non-empty. Notice that the set $\mathbb{G}$ (trivially) satisfies conditions (ia), (ib), and (ii) in Definition 4. This motivates the requirement of a minimality condition, namely condition (iii).

Theorem 2. A pairwise farsightedly stable set of networks exists.

Proof. Notice that $\mathbb{G}$ satisfies conditions (i) and (ii). Let us proceed by contradiction. Assume that there does not exist any set of networks $G \subseteq \mathbb{G}$ that is pairwise farsightedly stable. This means that for any $G^{0} \subseteq \mathbb{G}$ that satisfies conditions (i) and (ii) in Definition 4, we can find a proper subset $G^{1}$ that satisfies conditions (i) and (ii). Iterating this reasoning we can build an infinite decreasing sequence $\left\{G^{k}\right\}_{k} \geqslant 0$ of subsets of $\mathbb{G}$ satisfying conditions (i) and (ii). But since $\mathbb{G}$ has finite cardinality, this is not possible.

We show next that in Example 1 with $n=3$, the set consisting of the complete network is the unique pairwise farsightedly stable set whatever the fine $\phi$.

\footnotetext{
9 There are some random dynamic models of network formation that are based on incentives to form links such as Watts (2002), Jackson and Watts (2002), and Tercieux and Vannetelbosch (2006). These models aim to use the random process to select from the set of pairwise stable networks.
} 
We consider first the case $\phi<3 / 2$. It can be verified that $F\left(g_{0}\right)=\left\{g_{1}, g_{2}, g_{3}, g_{7}\right\}, F\left(g_{1}\right)=\left\{g_{2}, g_{3}, g_{7}\right\}, F\left(g_{2}\right)=$ $\left\{g_{1}, g_{3}, g_{7}\right\}, F\left(g_{3}\right)=\left\{g_{1}, g_{2}, g_{7}\right\}, F\left(g_{4}\right)=\left\{g_{1}, g_{2}, g_{3}, g_{7}\right\}, F\left(g_{5}\right)=\left\{g_{1}, g_{2}, g_{3}, g_{7}\right\}, F\left(g_{6}\right)=\left\{g_{1}, g_{2}, g_{3}, g_{7}\right\}$, and $F\left(g_{7}\right)=\emptyset$. Notice that the analysis of farsighted improving paths can be intricate. The only way to go from $g_{1}$ to $g_{2}$ is via $g_{4}$. At the same time it holds that $g_{4} \notin F\left(g_{1}\right)$. Indeed, players 1 and 2 make a link to go from $g_{1}$ to the intermediate network $g_{4}$ in the anticipation that player 3 will subsequently delete his link with player 1 . To go from $g_{1}$ to the terminal network $g_{4}$ is a strict deterioration for players 2 and 3 . The only thing player 1 can do is to sever his link with player 2 , which leads to $g_{0}$. This is not helpful for player 1 , since once at $g_{0}$ he is still the only one that is better off at $g_{4}$ compared to $g_{0}$, and there is nothing that he can do anymore.

We show next that $\left\{g_{7}\right\}$ is pairwise farsightedly stable. Since $g_{7} \in \bigcap_{g \in \mathbb{G} \backslash\left\{g_{7}\right\}} F(g)$, condition (ii) of the definition is clearly satisfied. Moreover, condition (i) is satisfied, since any deviation from $g_{7}$ may lead back to $g_{7}$. Clearly, $\left\{g_{7}\right\}$ is minimal, so condition (iii) is satisfied too.

There are no other pairwise farsightedly stable sets. Since $F\left(g_{7}\right)=\emptyset$, condition (ii) implies that $g_{7}$ belongs to any pairwise farsightedly stable set. Since $\left\{g_{7}\right\}$ is pairwise farsightedly stable, using condition (iii) it follows that $\left\{g_{7}\right\}$ is the only pairwise farsightedly stable set.

Take now $\phi \geqslant 3 / 2$. For $3 / 2 \leqslant \phi \leqslant 3$ we have $F\left(g_{0}\right)=\left\{g_{1}, g_{2}, g_{3}, g_{4}, g_{5}, g_{6}, g_{7}\right\}, F\left(g_{1}\right)=\left\{g_{4}, g_{5}, g_{7}\right\}, F\left(g_{2}\right)=$ $\left\{g_{4}, g_{6}, g_{7}\right\}, F\left(g_{3}\right)=\left\{g_{5}, g_{6}, g_{7}\right\}, F\left(g_{4}\right)=\left\{g_{7}\right\}, F\left(g_{5}\right)=\left\{g_{7}\right\}, F\left(g_{6}\right)=\left\{g_{7}\right\}$, and $F\left(g_{7}\right)=\emptyset$. For $\phi>3$ we have $F\left(g_{0}\right)=$ $\left\{g_{1}, g_{2}, g_{3}, g_{4}, g_{5}, g_{6}, g_{7}\right\}, F\left(g_{1}\right)=\left\{g_{4}, g_{5}, g_{6}, g_{7}\right\}, F\left(g_{2}\right)=\left\{g_{4}, g_{5}, g_{6}, g_{7}\right\}, F\left(g_{3}\right)=\left\{g_{4}, g_{5}, g_{6}, g_{7}\right\}, F\left(g_{4}\right)=\left\{g_{7}\right\}, F\left(g_{5}\right)=$ $\left\{g_{7}\right\}, F\left(g_{6}\right)=\left\{g_{7}\right\}$, and $F\left(g_{7}\right)=\emptyset$. So, $g_{7} \in \bigcap g \in \mathbb{G} \backslash\left\{g_{7}\right\} F(g)$ for $\phi \geqslant 3 / 2$. Since $F\left(g_{7}\right)=\emptyset$, we can use the same arguments as in the case $\phi<3 / 2$ and can therefore conclude that $\left\{g_{7}\right\}$ is the unique pairwise farsightedly stable set.

\section{Characterizations of pairwise farsightedly stable sets}

The next theorem provides an easy to verify condition for a set $G$ to be pairwise farsightedly stable.

Theorem 3. If for every $g^{\prime} \in \mathbb{G} \backslash G$ we have $F\left(g^{\prime}\right) \cap G \neq \emptyset$ and for every $g \in G, F(g) \cap G=\emptyset$, then $G$ is a pairwise farsightedly stable set.

Proof. Condition (ii) is trivially satisfied.

Suppose condition (i) is not satisfied. Then there is $g \in G$ and a deviation to $g^{\prime} \notin G$ such that every $g^{\prime \prime} \in F\left(g^{\prime}\right) \cap G$ defeats $g$. In particular, it then follows that $g^{\prime \prime} \in F(g)$, a contradiction, since by assumption there is no $g^{\prime \prime} \in G$ with that property. Consequently, condition (i) holds.

To verify condition (iii), suppose there is a proper subset $G^{\prime}$ of $G$ that satisfies conditions (i) and (ii). Let $g$ be in $G$ but not in $G^{\prime}$. Then $F(g) \cap G^{\prime} \subseteq F(g) \cap G=\emptyset$, where the equality follows by the assumption in the theorem. It follows that $G^{\prime}$ violates condition (ii), leading to a contradiction. We have shown that $G$ is minimal.

Later on in the paper, we will show by means of Example 3 that Theorem 3 cannot be extended to an "if and only if" statement. The "if and only if" statement is true, however, when restricting the scope of the theorem to sets consisting of a single network.

Theorem 4. The set $\{g\}$ is a pairwise farsightedly stable set if and only if for every $g^{\prime} \in \mathbb{G} \backslash\{g\}$ we have $g \in F\left(g^{\prime}\right)$.

Proof. If $\{g\}$ is a pairwise farsightedly stable set, then by condition (ii) in Definition 4 it follows that $g \in F\left(g^{\prime}\right)$ for every $g^{\prime} \in \mathbb{G} \backslash\{g\}$.

Now suppose that for every $g^{\prime} \in \mathbb{G} \backslash\{g\}$ we have $g \in F\left(g^{\prime}\right)$. Condition (ii) is trivially satisfied. Since $g \in F(g+i j)$ and $g \in F(g-i j)$, conditions (ia) and (ib) hold. Finally, condition (iii) is satisfied because $\{g\}$ is a singleton.

Theorem 4 tells us that $\{g\}$ is a pairwise farsightedly stable set if and only if there exists a farsighted improving path from any network leading to $g$. Condition (iii) of the definition implies that if $\{g\}$ is a pairwise farsightedly stable set, then $g$ does not belong to any other pairwise farsightedly stable set. But there may be pairwise farsightedly stable sets not containing $g$.

The next result provides a full characterization for unique pairwise farsightedly stable sets.

Theorem 5. The set $G$ is the unique pairwise farsightedly stable set if and only if $G=\{g \in \mathbb{G} \mid F(g)=\emptyset\}$ and for every $g^{\prime} \in \mathbb{G} \backslash G$, $F\left(g^{\prime}\right) \cap G \neq \varnothing$.

Proof. $(\Leftarrow)$ Condition (ii) of Definition 4 is trivially satisfied. Suppose condition (i) is not satisfied. Then there is $g \in G$ and $i j \notin g$ such that $g+i j \notin G$ and for every $g^{\prime} \in F(g+i j) \cap G$ it holds that $\left(Y_{i}\left(g^{\prime}, v\right), Y_{j}\left(g^{\prime}, v\right)\right)>\left(Y_{i}(g, v), Y_{j}(g, v)\right)$, or there is $g \in G$ and $i j \in g$ such that $g-i j \notin G$ and for every $g^{\prime} \in F(g+i j) \cap G$ it holds that $Y_{i}\left(g^{\prime}, v\right)>Y_{i}(g, v)$. In both cases it follows that $g^{\prime} \in F(g)$, a contradiction, since by assumption $F(g)=\emptyset$. Consequently, condition (i) holds. Since for every 
$g \in G, F(g)=\emptyset$, by condition (ii) it holds that $G$ is a subset of any pairwise farsightedly stable set. It then follows from condition (iii) that $G$ is the unique pairwise farsightedly stable set.

$(\Rightarrow)$ Condition (ii) yields that for every $g^{\prime} \in \mathbb{G} \backslash G, F\left(g^{\prime}\right) \cap G \neq \emptyset$. From this it also follows that every $g$ with $F(g)=\emptyset$ belongs to $G$. It remains to be shown that for every $g \in G, F(g)=\emptyset$. Suppose not. Let $g^{*}$ and $g^{\prime}$ be such that $g^{*} \in G$ and $g^{\prime} \in F\left(g^{*}\right)$. Consider $G^{\prime}=\left\{g^{\prime}\right\} \cup\left\{g \in \mathbb{G} \mid g^{\prime} \notin F(g)\right\}$. Notice that $g^{*} \notin G^{\prime}$ and that for any $g \notin G^{\prime}$ we have that $g^{\prime} \in F(g)$.

Claim: $G^{\prime}$ satisfies conditions (i) and (ii).

Since for any $g \notin G^{\prime}$ we have that $g^{\prime} \in F(g)$, condition (ii) is satisfied. Consider any pairwise deviation from $g^{\prime}$ to $g^{\prime \prime} \notin G^{\prime}$. By construction of $G^{\prime}, g^{\prime} \in F\left(g^{\prime \prime}\right)$ and the deviation is deterred. Consider any pairwise deviation from any $g^{0} \in G^{\prime} \backslash\left\{g^{\prime}\right\}$ to some $g^{\prime \prime} \notin G^{\prime}$. Suppose that all $g \in F\left(g^{\prime \prime}\right) \cap G^{\prime}$ are preferred by the player(s) initially deviating from $g^{0}$. Then it follows that $F\left(g^{\prime \prime}\right) \cap G^{\prime} \subseteq F\left(g^{0}\right)$. By definition of $G^{\prime}, g^{\prime} \in F\left(g^{\prime \prime}\right)$, so $g^{\prime} \in F\left(g^{\prime \prime}\right) \cap G^{\prime} \subseteq F\left(g^{0}\right)$, contradicting $g^{\prime} \notin F\left(g^{0}\right)$ for any $g^{0} \in G^{\prime} \backslash\left\{g^{\prime}\right\}$. Consequently, all pairwise deviations from $g^{0} \in G^{\prime} \backslash\left\{g^{\prime}\right\}$ are deterred. Since we already showed that pairwise deviations from $g^{\prime}$ are deterred too, the set $G^{\prime}$ satisfies condition (i).

Finally, if $G^{\prime}$ satisfies condition (iii), then $G^{\prime}$ is a pairwise farsightedly stable set, a contradiction to the uniqueness of $G$. If $G^{\prime}$ does not satisfy condition (iii), then, following the reasoning in the proof of Theorem 2, there is a proper subset $G^{\prime \prime}$ of $G^{\prime}$ satisfying conditions (i)-(iii). Since $g^{*} \in G \backslash G^{\prime \prime}$, it holds that $G \neq G^{\prime \prime}$ and we obtain a contradiction to the uniqueness of $G$.

Theorem 5 implies that if $G$ is the unique pairwise farsightedly stable set and the network $g$ belongs to $G$, then $F(g)=\emptyset$, which implies that $g$ is pairwise stable. Thus, pairwise farsighted stability is a refinement of pairwise stability when there is a unique pairwise farsightedly stable set.

From Theorem 5 we obtain the following corollary that provides the necessary and sufficient conditions such that there is a unique pairwise farsightedly stable set consisting of a single network.

Corollary 1. The set $\{g\}$ is the unique pairwise farsightedly stable set if and only if for every $g^{\prime} \in \mathbb{G} \backslash\{g\}$ we have $g \in F\left(g^{\prime}\right)$ and $F(g)=\emptyset$.

If for every $g^{\prime} \in \mathbb{G} \backslash\{g\}$ we have $g \in F\left(g^{\prime}\right)$, then by Theorem $4\{g\}$ is a pairwise farsightedly stable set. If, moreover, $F(g)=\emptyset$, then $\{g\}$ is the unique pairwise farsightedly stable set by Corollary 1 . If, on the other hand, $F(g) \neq \emptyset$, then there is another pairwise farsightedly stable set by Corollary 1 .

Using Theorem 4 we prove that in the example of criminal networks with $n$ players, the complete network $\left\{g^{N}\right\}$ is a pairwise farsightedly stable set.

Proposition 1. In the criminal networks model, the set $\left\{g^{N}\right\}$ is a pairwise farsightedly stable set.

Proof. We show that for every $g \in \mathbb{G} \backslash\left\{g^{N}\right\}$ we have $g^{N} \in F(g)$. (1) We show that from any $g \neq g^{N}$ there is always a player who wants to delete a link looking forward to $g^{N}$. This enables us to build a sequence of networks where at each step some player (who is looking forward to $g^{N}$ ) is deleting a link until we reach the empty network. (2) Next, starting from the empty network, we build up a sequence of networks towards $g^{N}$ so that, at each step, links are only added, and players that are adding links are strictly better off at $g^{N}$ compared to the current network. Then, (1) and (2) implies that from any possible network there is a farsighted improving path leading to the complete network $g^{N}$.

In the complete network we have that $Y_{i}\left(g^{N}\right)=y_{i}\left(g^{N}\right)=B / n$ because $p_{i}\left(g^{N}\right)=0$ for all $i \in N$. Notice that expected payoffs of members of a component do not depend on how other components are structured (are linked). They only depend on the number of links and on the number of criminals within the component. That is, criminal $i$ who belongs to group $S \in \Pi(g)$ will get $Y_{i}(g)=y_{i}(g)\left[1-p_{i}(g) \phi\right]=(|S| / n) \alpha_{i}(g) B\left[1-\left(n-1-n_{i}\right) \phi / n\right]$, with $\alpha_{i}(g)=\left[\#\left\{j \in S \mid n_{j}=\bar{n}(S)\right\}\right]^{-1}$ if $n_{i}=\bar{n}(S)$ and $\alpha_{i}(g)=0$ otherwise.

(1) Take any $g \neq g^{N}$. Case 1: For all $S \in \Pi(g)$, for every $i, j \in S$, we have that $n_{i}=n_{j}$. Then, for every $i \in N, Y_{i}(g)=$ $B / n\left[1-\left(n-1-n_{i}\right) \phi / n\right]<B / n=Y_{i}\left(g^{N}\right)$ and thus everyone who has a link is willing to delete a link looking forward to $g^{N}$. Case 2: There exists $S \in \Pi(g)$ such that $n_{i}<\bar{n}(S)$. Then, player $i$ gets $Y_{i}(g)=0$, so $i$ wants to delete a link looking forward to $g^{N}$. We can repeat the arguments of Cases 1 and 2 until we reach the empty network.

(2) Once we have reached the empty network we build up a sequence of networks towards $g^{N}$ as follows. For $k=$ $1, \ldots, n$, we successively build networks so that the subset of players $\{1, \ldots, k\}$ forms a complete component, and players $k+1, \ldots, n$ are singletons (do not have any link). We start with the empty network denoted $g^{1}$. Adding to $g^{1}$ the link $\{1,2\}$ leads to the network $g^{2}$. Notice that $\{1,2\}$ is a complete component of $g^{2}$, whereas players $3, \ldots, n$ are singletons. Let $g^{k}$, for some $k \in\{1, \ldots, n\}$, be a network such that the subset of players $\{1, \ldots, k\}$ forms a complete component, and players $k+1, \ldots, n$ are singletons. To $g^{k}$ we add successively the links $\{1, k+1\},\{2, k+1\}, \ldots,\{k, k+1\}$ to obtain the network $g^{k+1}$. Along this sequence of networks, the players that are adding a link are strictly better off at $g^{N}$ compared to what they obtain at the current network. Indeed, when involved in adding a link, player 1 has a payoff of $B / n[1-(n-k) \phi / n]<B / n$, player $k+1$ has a payoff of $B / n[1-(n-1) \phi / n]<B / n$ (before linking to player 1 ) or 0 (before linking to players $\{2, \ldots, k\}$ ), and the other players have a payoff of 0 .

Thus, for all $g \neq g^{N}$ we have $g^{N} \in F(g)$. Applying Theorem 4, we conclude that $\left\{g^{N}\right\}$ is a pairwise farsightedly stable set. 


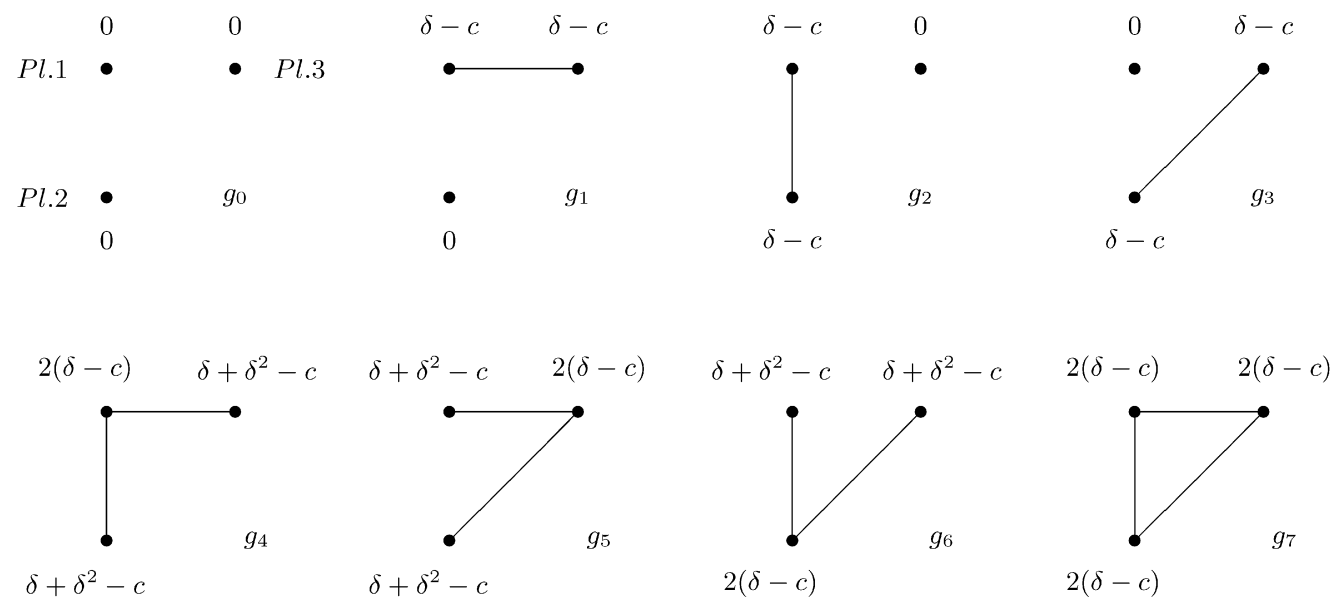

Fig. 2. The symmetric connections model with three players.

\section{The symmetric connections model and the co-author model}

Example 2 (Symmetric connections model). (See Jackson and Wolinsky (1996).) Players form links with each other in order to exchange information. If player $i$ is connected to player $j$ by a path of $t$ links, then player $i$ receives a payoff of $\delta^{t}$ from his indirect connection with player $j$. It is assumed that $0<\delta<1$, and so the payoff $\delta^{t}$ decreases as the path connecting players $i$ and $j$ increases; thus information that travels a long distance becomes diluted and is less valuable than information obtained from a closer neighbor. Each direct link $i j$ results in a cost $c$ to both $i$ and $j$. This cost can be interpreted as the time a player must spend with another player in order to maintain a direct link. Player $i$ 's payoff from a network $g$ is given by

$$
Y_{i}(g)=\sum_{j \neq i} \delta^{t(i j)}-\sum_{j: i j \in g} c,
$$

where $t(i j)$ is the number of links in the shortest path between $i$ and $j$ (setting $t(i j)=\infty$ if there is no path between $i$ and $j)$.

In Fig. 2 we have depicted the 3-player case where (i) for $c<\delta(1-\delta)$, the complete network ( $g_{7}$ in Fig. 2) is the unique pairwise stable network and $\left\{g_{7}\right\}$ is the pairwise myopically stable set, (ii) for $\delta(1-\delta)<c<\delta$, the star networks $\left(g_{4}, g_{5}, g_{6}\right.$ in Fig. 2) are pairwise stable and $\left\{g_{4}, g_{5}, g_{6}\right\}$ is the pairwise myopically stable set, and (iii) for $c>\delta$, the empty network is the unique pairwise stable network and $\left\{g_{0}\right\}$ is the pairwise myopically stable set.

Applying our concept of pairwise farsightedly stable sets to the symmetric connections model with three players, we obtain that a network $g$ is pairwise stable if and only if $\{g\}$ is a pairwise farsightedly stable set. First we consider the case $c<\delta(1-\delta)$. It holds that $F\left(g_{0}\right)=\left\{g_{1}, g_{2}, g_{3}, g_{4}, g_{5}, g_{6}, g_{7}\right\}, F\left(g_{1}\right)=\left\{g_{4}, g_{5}, g_{6}, g_{7}\right\}, F\left(g_{2}\right)=\left\{g_{4}, g_{5}, g_{6}, g_{7}\right\}, F\left(g_{3}\right)=$ $\left\{g_{4}, g_{5}, g_{6}, g_{7}\right\}, F\left(g_{4}\right)=\left\{g_{5}, g_{6}, g_{7}\right\}, F\left(g_{5}\right)=\left\{g_{4}, g_{6}, g_{7}\right\}, F\left(g_{6}\right)=\left\{g_{4}, g_{5}, g_{7}\right\}$, and $F\left(g_{7}\right)=\emptyset$. Now it follows by Corollary 1 that $\left\{g_{7}\right\}$ is the unique pairwise farsightedly stable set.

Next we consider the case $\delta(1-\delta)<c<\delta$. It holds that $F\left(g_{0}\right)=\left\{g_{1}, g_{2}, g_{3}, g_{4}, g_{5}, g_{6}\right\}, F\left(g_{1}\right)=\left\{g_{4}, g_{5}, g_{6}\right\}, F\left(g_{2}\right)=$ $\left\{g_{4}, g_{5}, g_{6}\right\}, F\left(g_{3}\right)=\left\{g_{4}, g_{5}, g_{6}\right\}, F\left(g_{4}\right)=\left\{g_{5}, g_{6}\right\}, F\left(g_{5}\right)=\left\{g_{4}, g_{6}\right\}, F\left(g_{6}\right)=\left\{g_{4}, g_{5}\right\}$, and $F\left(g_{7}\right)=\left\{g_{4}, g_{5}, g_{6}\right\}$.

By a repeated application of Theorem 4 , it follows that $\left\{g_{4}\right\},\left\{g_{5}\right\}$, and $\left\{g_{6}\right\}$ are pairwise farsightedly stable sets.

Finally, we examine the case $c>\delta$. One may verify that $F\left(g_{0}\right)=\emptyset, F\left(g_{1}\right)=\left\{g_{0}\right\}, F\left(g_{2}\right)=\left\{g_{0}\right\}, F\left(g_{3}\right)=\left\{g_{0}\right\}, F\left(g_{4}\right)=$ $\left\{g_{0}, g_{1}, g_{2}\right\}, F\left(g_{5}\right)=\left\{g_{0}, g_{1}, g_{3}\right\}, F\left(g_{6}\right)=\left\{g_{0}, g_{2}, g_{3}\right\}$, and $F\left(g_{7}\right)=\left\{g_{0}, g_{1}, g_{2}, g_{3}, g_{4}, g_{5}, g_{6}\right\}$. It follows by Corollary 1 that $\left\{g_{0}\right\}$ is the unique pairwise farsightedly stable set.

Example 3 (Co-author model). (See Jackson and Wolinsky (1996).) Each player is a researcher who spends time writing papers. If two players are connected, then they are working on a paper together. The amount of time researcher $i$ spends on a given project is inversely related to the number of projects, $n_{i}$, that he is involved in. Formally, player $i$ 's payoff is given by

$$
Y_{i}(g)=\sum_{j: i j \in g}\left(\frac{1}{n_{i}}+\frac{1}{n_{j}}+\frac{1}{n_{i} n_{j}}\right)
$$

for $n_{i}>0$. For $n_{i}=0$ we assume that $Y_{i}(g)=0$. In Fig. 3 we have depicted the 3 -player case. It is easily verified that the complete network $g_{7}$ is the unique pairwise stable network. Moreover, it is easy to demonstrate that the pairwise myopically stable set is $\left\{g_{7}\right\}$. 


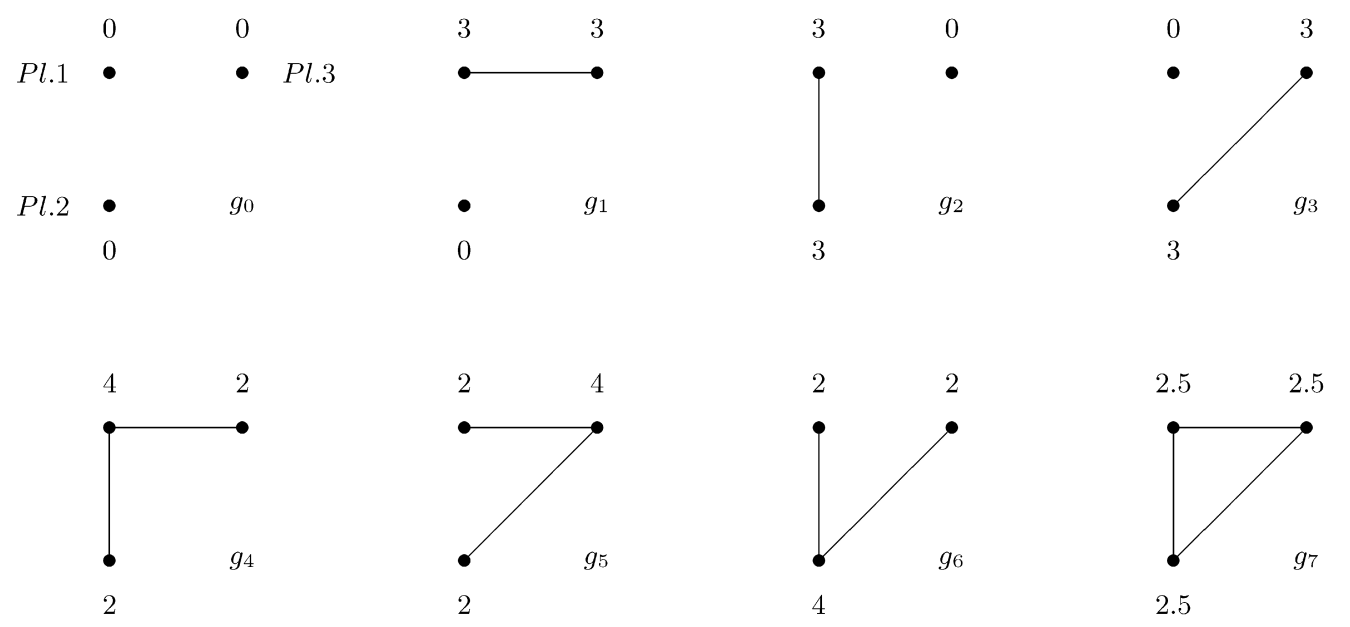

Fig. 3. The co-author model with three players.

No singleton set is pairwise farsightedly stable in Example 3. Indeed, there is no network such that there is a farsighted improving path from any other network leading to it. More precisely, $F\left(g_{0}\right)=\left\{g_{1}, g_{2}, g_{3}, g_{4}, g_{5}, g_{6}\right\}, F\left(g_{1}\right)=\left\{g_{4}, g_{5}\right\}$, $F\left(g_{2}\right)=\left\{g_{4}, g_{6}\right\}, F\left(g_{3}\right)=\left\{g_{5}, g_{6}\right\}, F\left(g_{4}\right)=\left\{g_{7}\right\}, F\left(g_{5}\right)=\left\{g_{7}\right\}, F\left(g_{6}\right)=\left\{g_{7}\right\}$, and $F\left(g_{7}\right)=\emptyset$. However, a set formed by the complete and two star networks is a pairwise farsightedly stable set of networks. The pairwise farsightedly stable sets are $\left\{g_{4}, g_{5}, g_{7}\right\},\left\{g_{4}, g_{6}, g_{7}\right\},\left\{g_{5}, g_{6}, g_{7}\right\}$, and $\left\{g_{1}, g_{2}, g_{3}, g_{7}\right\}$ in the co-author model with three players.

\section{Efficiency and farsighted stability}

We now turn to the question of the relationship between farsighted stability and efficiency of networks. A first result is that the set of pairwise farsightedly stable networks and the set of strongly efficient networks, those which are socially optimal, may be disjoint for all allocation rules that are component balanced and anonymous. ${ }^{10}$

Theorem 6. There exists a value function such that for every component balanced and anonymous rule, strongly efficient networks are not included in any of the pairwise farsightedly stable sets.

Proof. Take the following value function defined for any $g \in \mathbb{G}: v(\{12,13,23\})=9, v(\{12,13\})=0, v(\{12,23\})=0$, $v(\{13,23\})=0, v(\{12\})=8, v(\{13\})=8, v(\{23\})=8$, and $v(\emptyset)=0$. Fix any component balanced and anonymous allocation rule $Y$. Then, by component balance and anonymity,

(i) $Y_{1}(\{12,13,23\}, v)=Y_{2}(\{12,13,23\}, v)=Y_{3}(\{12,13,23\}, v)=3$,

(ii) $Y_{1}(\{12,23\}, v)=c, Y_{3}(\{12,23\}, v)=c, Y_{2}(\{12,23\}, v)=-2 c, Y_{2}(\{12,13\}, v)=c, Y_{3}(\{12,13\}, v)=c, Y_{1}(\{12,13\}, v)=$ $-2 c, Y_{1}(\{13,23\}, v)=c, Y_{2}(\{13,23\}, v)=c, Y_{3}(\{13,23\}, v)=-2 c$,

(iii) $Y_{1}(\{12\}, v)=Y_{2}(\{12\}, v)=4, Y_{3}(\{12\}, v)=0, Y_{1}(\{13\}, v)=Y_{3}(\{13\}, v)=4, Y_{2}(\{13\}, v)=0, Y_{2}(\{23\}, v)=Y_{3}(\{23\}, v)=$ $4, Y_{1}(\{23\}, v)=0$, and

(iv) $Y_{1}(\emptyset, v)=Y_{2}(\emptyset, v)=Y_{3}(\emptyset, v)=0$.

The unique strongly efficient network is $\{12,13,23\}$. We have:

(i) $F(\emptyset)=\{\{12\},\{13\},\{23\},\{12,13,23\}\}$;

(ii) $F(\{12\})=\{\{13\},\{23\}\}, F(\{13\})=\{\{12\},\{23\}\}, F(\{23\})=\{\{12\},\{13\}\}$;

(iii) For $c<3, F(\{12,13\})=\{\{12\},\{13\},\{23\},\{12,13,23\}\}$, for $3 \leqslant c<4, F(\{12,13\})=\{\{12\},\{13\}$, $\{23\}\}$, and for $c \geqslant 4$, $F(\{12,13\})=\{\{12\},\{13\}\}$. Next, for $c<3, F(\{12,23\})=\{\{12\},\{13\},\{23\},\{12,13,23\}\}$, for $3 \leqslant c<4, F(\{12,23\})=\{\{12\}$, $\{13\},\{23\}\}$, and for $c \geqslant 4, F(\{12,23\})=\{\{12\},\{23\}\}$. And, for $c<3, F(\{13,23\})=\{\{12\},\{13\},\{23\},\{12,13,23\}\}$, for $3 \leqslant c<4, F(\{13,23\})=\{\{12\},\{13\},\{23\}\}$, and for $c \geqslant 4, F(\{13,23\})=\{\{13\},\{23\}\}$;

(iv) For $c<3, F(\{12,13,23\})=\{\{12\},\{13\},\{23\},\{12,13\},\{12,23\},\{13,23\}\}$, for $c \geqslant 3, F(\{12,13,23\})=\{\{12\},\{13\},\{23\}\}$.

Thus, $\{\{12\}\},\{\{13\}\}$, and $\{\{23\}\}$ are the only pairwise farsightedly stable sets.

10 Bhattacharya (2005) has obtained a similar result with respect to the notion of the largest consistent set. 


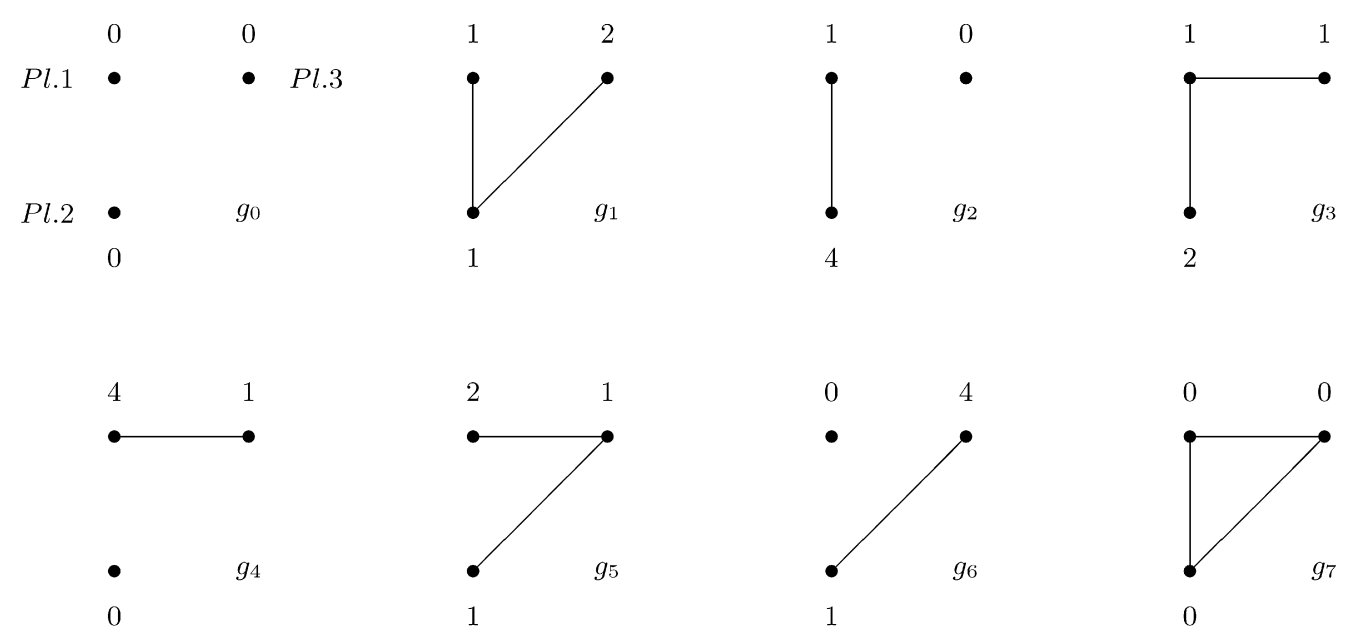

Fig. 4. Non-existence of von Neumann-Morgenstern pairwise farsightedly stable sets.

A second result considers the case where there is a network that strictly Pareto dominates all other networks. That is, if there is a network $g$ such that for all $g^{\prime} \in \mathbb{G} \backslash\{g\}$ it holds that, for all $i, Y_{i}(g, v)>Y_{i}\left(g^{\prime}, v\right)$. Although the network that strictly Pareto dominates all others is pairwise stable, there might be many more pairwise stable networks. We will show in Section 8 that also the concept of the largest pairwise consistent set suffers from a similar defect. The following result asserts that pairwise farsighted stability singles out the Pareto dominating network as the unique pairwise farsightedly stable set.

Theorem 7. If there is a network $g$ that strictly Pareto dominates all other networks, then $\{\mathrm{g}\}$ is the unique pairwise farsightedly stable set.

Proof. It is immediate that $g \in F\left(g^{\prime}\right)$ for all $g^{\prime} \in \mathbb{G} \backslash\{g\}$ and that $F(g)=\emptyset$. Corollary 1 leads to the desired result.

We next provide sufficient conditions on the allocation rule and/or the value function such that there is no conflict between strong efficiency and farsighted stability.

An immediate application of Theorem 7 is the case of increasing returns to link creation as defined in Dutta et al. (2005). This property requests that along every nested sequence of increasingly connected networks, there is a threshold network for which the value turns nonnegative, and both aggregate as well as payoffs of individuals who form extra links then increase as the network becomes even larger. Under this condition, and with a componentwise egalitarian allocation rule, $g^{N}$ Pareto dominates all other networks, so Theorem 7 applies.

An allocation rule is said to be egalitarian if for every $v \in \mathcal{V}$ and $g \in \mathbb{G}, Y_{i}(g, v)=v(g) / n$. The following result follows as a corollary to Theorem 7 .

Corollary 2. Suppose that $Y$ is the egalitarian rule and there is a unique strongly efficient network $g^{e}$. Then, $\left\{g^{e}\right\}$ is the unique pairwise farsightedly stable set.

\section{The von Neumann-Morgenstern pairwise farsightedly stable set}

The pairwise farsightedly stable set requires deterrence of external deviations, external stability, and minimality. The von Neumann-Morgenstern stable set (von Neumann and Morgenstern, 1944) imposes internal and external stability. Incorporating the notion of farsighted improving paths into the original definition of the von Neumann-Morgenstern stable set, we obtain the von Neumann-Morgenstern pairwise farsightedly stable set.

Definition 5. The set $G$ is a von Neumann-Morgenstern pairwise farsightedly stable set if (i) $\forall g \in G, F(g) \cap G=\emptyset$ and (ii) $\forall g^{\prime} \in \mathbb{G} \backslash G, F\left(g^{\prime}\right) \cap G \neq \emptyset$.

Von Neumann-Morgenstern pairwise farsightedly stable sets do not always exist. Consider the situation where three players can forms links and where the payoffs are given in Fig. 4. We have $F\left(g_{0}\right)=F\left(g_{7}\right)=\left\{g_{1}, g_{2}, g_{3}, g_{4}, g_{5}, g_{6}\right\}, F\left(g_{1}\right)=$ $\left\{g_{2}, g_{3}\right\}, F\left(g_{2}\right)=\left\{g_{3}, g_{4}, g_{5}\right\}, F\left(g_{3}\right)=\left\{g_{4}, g_{5}\right\}, F\left(g_{4}\right)=\left\{g_{1}, g_{5}, g_{6}\right\}, F\left(g_{5}\right)=\left\{g_{1}, g_{6}\right\}, F\left(g_{6}\right)=\left\{g_{1}, g_{2}, g_{3}\right\}$. We prove that there is no von Neumann-Morgenstern pairwise farsightedly stable set. Suppose on the contrary that $G$ is a von NeumannMorgenstern pairwise farsightedly stable set. Suppose $g_{1} \in G$. The internal stability condition implies that no other network 
can belong to $G$. Since $F\left(g_{2}\right) \cap\left\{g_{1}\right\}=\emptyset$ it follows that external stability is violated, a contradiction. As a consequence, $g_{1} \notin G$. A symmetric argument leads to the result that $g_{3} \notin G$ and $g_{5} \notin G$. Suppose now that $g_{2} \in G$. The internal stability condition implies that no other network can belong to $G$. Since $F\left(g_{5}\right) \cap\left\{g_{2}\right\}=\emptyset$ it follows that external stability is violated, a contradiction. By symmetry it follows that $g_{4} \notin G$ and $g_{6} \notin G$. Suppose $g_{0} \in G$. Internal stability implies that no other network can belong to $G$. Since $F\left(g_{1}\right) \cap\left\{g_{0}\right\}=\emptyset$, it follows that external stability is violated, a contradiction. By a similar argument we can show that $g_{7} \notin G$. The only remaining possibility is $G=\emptyset$. This clearly violates external stability. Thus, there is no von Neumann-Morgenstern pairwise farsightedly stable set in this example. However, pairwise farsightedly stable sets do exist by virtue of Theorem 2 . The pairwise farsightedly stable sets are $\left\{g_{1}, g_{2}, g_{3}\right\},\left\{g_{3}, g_{4}, g_{5}\right\}$, and $\left\{g_{1}, g_{5}, g_{6}\right\}$.

From Theorem 3 we immediately obtain the following corollary, which states that a von Neumann-Morgenstern pairwise farsightedly stable set is also a pairwise farsightedly stable set.

Corollary 3. If $G$ is a von Neumann-Morgenstern pairwise farsightedly stable set, then $G$ is a pairwise farsightedly stable set.

Since internal stability is automatically satisfied when a set of networks contains only one element, Theorem 4 leads to the following corollary.

Corollary 4. The set $\{g\}$ is a pairwise farsightedly stable set if and only if it is a von Neumann-Morgenstern pairwise farsightedly stable set.

From Theorem 5 and Corollary 3 we immediately get the next result, which implies the converse of Corollary 3, a unique pairwise farsightedly stable set is also a von Neumann-Morgenstern pairwise farsightedly stable set.

Corollary 5. If $G$ is the unique pairwise farsightedly stable set, then $G$ is the unique von Neumann-Morgenstern pairwise farsightedly stable set.

We have shown that replacing the internal stability condition in the von Neumann-Morgenstern pairwise farsightedly stable set by deterrence of external deviations and minimality, leads to a stability concept that contains the von NeumannMorgenstern pairwise farsightedly stable set, and is always non-empty.

Example 1 (Criminal networks, $n=3$ (continued)). Since $\left\{g_{7}\right\}$ is the unique pairwise farsightedly stable set, Corollary 4 shows that $\left\{g_{7}\right\}$ is the unique von Neumann-Morgenstern pairwise farsightedly stable set.

Example 3 (Co-author model (continued)). By Corollary 3 we have to analyze whether any of the pairwise farsightedly stable sets is a von Neumann-Morgenstern pairwise farsightedly stable set. The unique von Neumann-Morgenstern pairwise farsightedly stable set is given by $\left\{g_{1}, g_{2}, g_{3}, g_{7}\right\}$. The other pairwise farsightedly stable sets $\left\{g_{4}, g_{5}, g_{7}\right\},\left\{g_{4}, g_{6}, g_{7}\right\}$, $\left\{g_{5}, g_{6}, g_{7}\right\}$, are not von Neumann-Morgenstern pairwise farsightedly stable sets because they do not satisfy the internal stability condition.

\section{The largest pairwise consistent set}

In this section we study the relationship between pairwise farsighted stability and the largest pairwise consistent set, a concept that has been defined in Chwe (1994) for general social environments. By considering a network as a social environment, and by allowing only pairwise deviations, we obtain the definition of the largest pairwise consistent set.

Definition 6. $G$ is a pairwise consistent set if $\forall g \in G$,

(ia) $\forall i j \notin g, \exists g^{\prime} \in G$, where $g^{\prime}=g+i j$ or $g^{\prime} \in F(g+i j) \cap G$, such that $Y_{i}\left(g^{\prime}, v\right)<Y_{i}(g, v)$ or $Y_{j}\left(g^{\prime}, v\right)<Y_{j}(g, v)$ or $\left(Y_{i}\left(g^{\prime}, v\right), Y_{j}\left(g^{\prime}, v\right)\right)=\left(Y_{i}(g, v), Y_{j}(g, v)\right)$,

(ib) $\forall i j \in g$, $\exists g^{\prime}, g^{\prime \prime} \in G$, where $g^{\prime}=g-i j$ or $g^{\prime} \in F(g-i j) \cap G$, and $g^{\prime \prime}=g-i j$ or $g^{\prime \prime} \in F(g-i j) \cap G$, such that $Y_{i}\left(g^{\prime}, v\right) \leqslant$ $Y_{i}(g, v)$ and $Y_{j}\left(g^{\prime \prime}, v\right) \leqslant Y_{j}(g, v)$.

The largest pairwise consistent set is the pairwise consistent set that contains any pairwise consistent set.

The set $G$ is a pairwise consistent set if both external and internal deviations are deterred. The largest pairwise consistent set is the set that contains any pairwise consistent set. It follows from the results in Chwe (1994) that the largest pairwise consistent set exists, is non-empty, and satisfies external stability.

Our pairwise farsightedly stable sets need not be consistent in the sense of Chwe (1994) since we do not require internal deviations to be deterred. Moreover a pairwise consistent set does not necessarily satisfies the external stability condition. Only the largest pairwise consistent set is guaranteed to satisfy external stability.

Whenever $G$ is a von Neumann-Morgenstern pairwise farsightedly stable set, $G$ is also a pairwise farsightedly stable set (Corollary 3) and a largest pairwise consistent set (see Chwe, 1994). Replacing the internal and external stability conditions 
of the von Neumann-Morgenstern pairwise farsightedly stable set by the conditions that internal and external deviations should be deterred, Chwe (1994) has proposed a stability concept that always exists and that contains the von NeumannMorgenstern pairwise farsightedly stable set. In this paper, replacing the internal stability condition by the condition that external deviations should be deterred and the minimality condition, we propose another stability concept that also contains the von Neumann-Morgenstern pairwise farsightedly stable set.

Chwe (1994) provides the following iterative procedure to find the largest consistent set. Let $Z^{0} \equiv \mathbb{G}$. Then, $Z^{k}(k=$ $1,2, \ldots)$ is inductively defined as follows: $g \in Z^{k-1}$ belongs to $Z^{k}$ with respect to $Y$ and $v$ if

(ia) $\forall i j \notin g, \exists g^{\prime} \in Z^{k-1}$, where $g^{\prime}=g+i j$ or $g^{\prime} \in F(g+i j)$ such that $Y_{i}\left(g^{\prime}, v\right)<Y_{i}(g, v)$ or $Y_{j}\left(g^{\prime}, v\right)<Y_{j}(g, v)$ or $\left(Y_{i}\left(g^{\prime}, v\right), Y_{j}\left(g^{\prime}, v\right)\right)=\left(Y_{i}(g, v), Y_{j}(g, v)\right)$.

(ib) $\forall i j \in g, \exists g^{\prime}, g^{\prime \prime} \in Z^{k-1}$, where $g^{\prime}=g-i j$ or $g^{\prime} \in F(g-i j)$, and $g^{\prime \prime}=g-i j$ or $g^{\prime \prime} \in F(g-i j)$, such that $Y_{i}\left(g^{\prime}, v\right) \leqslant Y_{i}(g, v)$ and $Y_{j}\left(g^{\prime \prime}, v\right) \leqslant Y_{j}(g, v)$.

The largest pairwise consistent set is given by $\bigcap_{k \geqslant 1} Z^{k}$. That is, a network $g \in Z^{k-1}$ is stable (at step $k$ ) and belongs to $Z^{k}$, if all possible pairwise deviations are deterred. Consider a pairwise deviation from $g$ that involves making the link $i j$. There might be further pairwise deviations which end up at $g^{\prime}$, where $g+i j \rightarrow g^{\prime}$. If either $i$ or $j$ is worse off at $g^{\prime}$ or both are equally well off compared to the original network $g$, then the pairwise deviation is deterred. Similarly for a pairwise deviation from $g$ that involves deleting the link $i j$. There might be further pairwise deviations which end up at $g^{\prime}$ and $g^{\prime \prime}$ where $g-i j \rightarrow g^{\prime}$ and $g-i j \rightarrow g^{\prime \prime}$. If $i$ is equally well or worse off at $g^{\prime}$ and $j$ is equally well or worse off at $g^{\prime \prime}$ compared to the original network $g$, then the pairwise deviation is deterred. Since $\mathbb{G}$ is finite, there exists $m \in \mathbb{N}$ such that $Z^{k}=Z^{m}$ for all $k \geqslant m$, and $Z^{m}$ is the largest pairwise consistent set.

The next result states that if a network is not in the largest pairwise consistent set, it cannot be a pairwise farsightedly stable set of networks.

Theorem 8. If $\{g\}$ is a pairwise farsightedly stable set, then $g$ belongs to the largest pairwise consistent set.

Proof. Since $\{g\}$ is a pairwise farsightedly stable set we have that for all $i j \notin g: g \in F(g+i j)$ and for all $i j \in g: g \in F(g-i j)$. So $g \in Z^{1}$. By induction, $g \in Z^{k}$ for $k \geqslant 1$. So, $g$ belongs to the largest pairwise consistent set.

Remember that two networks $g$ and $g^{\prime}$ are adjacent if they differ by one link. The value function $v$ and allocation rule $Y$ exhibit no indifference if for any $g$ and $g^{\prime}$ that are adjacent either $g$ defeats $g^{\prime}$ or $g^{\prime}$ defeats $g$.

Theorem 9. Suppose that $Y$ and $v$ exhibit no indifference. If $g$ is pairwise stable then it belongs to the largest pairwise consistent set.

Proof. Since $Y$ and $v$ exhibit no indifference, we have that a pairwise stable network $g$ defeats (i) $g+i j$ for all $i j \notin g$ and (ii) $g-i j$ for all $i j \in g$. Thus, $g \in F(g+i j)$ and $g \in F(g-i j)$. So $g \in Z^{1}$. By induction $g \in Z^{k}$ for $k \geqslant 1$. So, $g$ belongs to the largest pairwise consistent set.

We claimed in Section 7 that even if there is a network that strictly Pareto dominates all other networks, the largest pairwise consistent set may contain other networks. It is not difficult to construct examples where the no indifference property holds, and some network strictly Pareto dominates all others. Moreover, such an example can be constructed such that inefficient networks are pairwise stable. It then follows from Theorem 9 that such a network also belongs to the largest pairwise consistent set. By virtue of Theorem 7, such a network does not belong to any pairwise farsightedly stable set.

Let us calculate the largest pairwise consistent set in two examples.

Example 1 (Criminal networks, $n=3, \phi<3 / 2$ (continued)). We apply the iterative procedure of Chwe (1994) to find the largest pairwise consistent set. We start with $Z^{0}=\left\{g_{0}, g_{1}, \ldots, g_{7}\right\}$.

Starting in $g_{0}$, players 1 and 2 can add the link $\{1,2\}$ to move to $g_{2}$. The indirect dominance relation implies that from there it is only possible to end up in $g_{1}, g_{3}$, or $g_{7}$. In all these networks both players have at least the same payoffs as in $g_{0}$, and at least one player has strictly higher payoffs. It follows that $g_{0} \notin Z^{1}$. Any deviation from $g_{1}$ may lead back to $g_{1}$ and is thereby deterred, so $g_{1} \in Z^{1}$. By symmetry it holds that $g_{2}, g_{3} \in Z^{1}$. Starting in $g_{4}$, players 2 and 3 can add the link $\{2,3\}$, resulting in $g_{7}$. Since $F\left(g_{7}\right)=\emptyset$ and both players have higher payoffs in $g_{7}$ than in $g_{4}$, we find that $g_{4} \notin Z^{1}$. By symmetry it holds that $g_{5}, g_{6} \notin Z^{1}$. Any deviation from $g_{7}$ may lead back to $g_{7}$ and is thereby deterred, so $g_{7} \in Z^{1}$. The same arguments can be used to show that $Z^{2}=Z^{1}$. It follows that the largest pairwise consistent set equals $\left\{g_{1}, g_{2}, g_{3}, g_{7}\right\}$.

Theorem 8 implies that $g_{7}$ belongs to the largest pairwise consistent set. This example therefore demonstrates that the largest pairwise consistent set may contain other networks too.

Example 3 (Co-author model (continued)). We apply the iterative procedure of Chwe (1994) to find the largest pairwise consistent set. We start with $Z^{0}=\left\{g_{0}, g_{1}, \ldots, g_{7}\right\}$. Starting in $g_{0}$, players 1 and 2 can add the link $\{1,2\}$ to move to $g_{2}$. The 
Table 1

The (no)-relationships among solution concepts for network stability.

\begin{tabular}{lll}
\hline Concept & Example 1 & Example 3 \\
\hline Pairwise myopically stable set & $\left\{g_{1}, g_{2}, g_{3}, g_{7}\right\}$ & $\left\{g_{7}\right\}$ \\
Pairwise farsightedly stable set & $\left\{g_{7}\right\}$ & $\left\{g_{4}, g_{5}, g_{7}\right\},\left\{g_{4}, g_{6,} g_{7}\right\},\left\{g_{5}, g_{6}, g_{7}\right\},\left\{g_{1}, g_{2}, g_{3}, g_{7}\right\}$ \\
vN-M farsighted stable set & $\left\{g_{7}\right\}$ & $\left\{g_{1}, g_{2}, g_{3}, g_{7}\right\}$ \\
Largest pairwise consistent set & $\left\{g_{1}, g_{2}, g_{3}, g_{7}\right\}$ & $\left\{g_{1}, g_{2}, g_{3}, g_{7}\right\}$ \\
\hline
\end{tabular}

indirect dominance relation implies that from there it is only possible to reach $g_{4}$ or $g_{6}$. In all these networks, players 1 and 2 have higher payoffs than at $g_{0}$. It follows that $g_{0} \notin Z^{1}$. Starting in $g_{4}$, players 2 and 3 will add a link to move to $g_{7}$. Since $F\left(g_{7}\right)=\emptyset$, no further moves will occur. Players 2 and 3 have higher payoffs at $g_{7}$ than at $g_{4}$. It follows that $g_{4} \notin Z^{1}$. For similar reasons, $g_{5} \notin Z^{1}$ and $g_{6} \notin Z^{1}$. It can be verified that $Z^{1}=\left\{g_{1}, g_{2}, g_{3}, g_{7}\right\}$.

We show next that $Z^{2}=\left\{g_{1}, g_{2}, g_{3}, g_{7}\right\}$. Starting in $g_{1}$, players 1 and 2 may add a link to go to $g_{4}$, a network not in $Z^{1}$. From $g_{4}$ the indirect dominance relation dictates a move to $g_{7}$. In $g_{7}$ player 1 is worse off than in $g_{1}$. It follows that no link will be added by them to $g_{1}$. Repeating such arguments, it can be shown that $Z^{2}=\left\{g_{1}, g_{2}, g_{3}, g_{7}\right\}=Z^{k}$ for all $k \geqslant 2$. It follows that the largest pairwise consistent set equals $\left\{g_{1}, g_{2}, g_{3}, g_{7}\right\}$.

Since the assumptions of Theorem 9 are satisfied in this example, it follows that $g_{7}$ belongs to the largest pairwise consistent set. The example shows that the largest pairwise consistent set may contain other networks too.

Table 1 summarizes our findings in Example 1 with $n=3$ and $\phi<3 / 2$ and Example 3.

\section{Conclusion}

We have proposed a new concept, the pairwise farsightedly stable set, to predict which networks may be formed among farsighted players. A set of networks $G$ is pairwise farsightedly stable (i) if all possible pairwise deviations from any network $g \in G$ to a network outside $G$ are deterred by the threat of ending worse off or equally well off, (ii) if there exists a farsighted improving path from any network outside the set leading to some network in the set, and (iii) if there is no proper subset of $G$ satisfying conditions (i) and (ii). We have shown that a pairwise farsightedly stable set always exists and we provide a full characterization of unique pairwise farsightedly stable sets of networks. As a corollary we have given the necessary and sufficient condition such that a unique pairwise farsightedly stable set consisting of a single network exists. We have found that the pairwise farsightedly stable sets and the set of strongly efficient networks may be disjoint. Nevertheless, contrary to other pairwise concepts, if there is a network that Pareto dominates all other networks, then that network is the unique prediction of pairwise farsighted stability. We have also been able to provide some conditions on the allocation rule and the value function such that pairwise farsighted stability singles out the strongly efficient network. Finally, we have studied the relationship between pairwise farsighted stability and other concepts such as pairwise myopic stability, the von Neumann-Morgenstern pairwise farsightedly stable set, and the largest pairwise consistent set. When there is a unique pairwise farsightedly stable set, then its elements are also pairwise stable. Pairwise stable networks belong to the largest pairwise consistent set when a mild no indifference criterion is satisfied. Moreover, any von Neumann-Morgenstern pairwise farsightedly stable set is also a pairwise farsightedly stable set. A pairwise farsightedly stable set consisting of a unique element is also a von Neumann-Morgenstern pairwise farsightedly stable set. If there is a unique pairwise farsightedly stable set, then it is also the unique von Neumann-Morgenstern pairwise farsightedly stable set. By means of examples we have shown that there is no general relationship between (i) pairwise farsightedly stable sets and pairwise myopically stable sets and (ii) pairwise farsightedly stable sets and largest pairwise consistent sets.

\section{Acknowledgments}

We would like to thank two anonymous referees for helpful comments. P. Jean-Jacques Herings would like to thank the Netherlands Organization for Scientific Research (NWO) for financial support. Vincent Vannetelbosch and Ana Mauleon are Research Associates of the National Fund for Scientific Research (FNRS). Vincent Vannetelbosch is Associate Fellow of CEREC, Facultés Universitaires Saint-Louis. Financial support from Spanish Ministerio de Educacion y Ciencia under the project SEJ 2006-06309/ECON, support from the Belgian French Community's program Action de Recherches Concertée 03/08-302 and 05/10-331 (UCL) and support of a SSTC grant from the Belgian Federal government under the IAP contract P6/09 are gratefully acknowledged.

\section{References}

Aumann, R., Myerson, R., 1988. Endogenous formation of links between players and coalitions: An application of the Shapley value. In: Roth, A. (Ed.), The Shapley Value. Cambridge University Press, Cambridge, pp. 175-191.

Bhattacharya, A., 2005. Stable and efficient networks with farsighted players: The largest consistent set. Mimeo. University of York.

Calvó-Armengol, A., Zenou, Y., 2004. Social networks and crime decisions: The role of social structure in facilitating delinquent behavior. Int. Econ. Rev. 45, 939-958.

Chwe, M.S., 1994. Farsighted coalitional stability. J. Econ. Theory 63, 299-325. 
Dutta, B., Mutuswami, S., 1997. Stable networks. J. Econ. Theory 76, 322-344.

Dutta, B., Ghosal, S., Ray, D., 2005. Farsighted network formation. J. Econ. Theory 122, 143-164.

Herings, P.J.J., Mauleon, A., Vannetelbosch, V., 2004. Rationalizability for social environments. Games Econ. Behav. 49, 135-156.

Jackson, M.O., 2003. The stability and efficiency of economic and social networks. In: Dutta, B., Jackson, M.O. (Eds.), Networks and Groups: Models of Strategic Formation. Springer-Verlag, Heidelberg, pp. 99-140.

Jackson, M.O., 2005. A survey of models of network formation: Stability and efficiency. In: Demange, G., Wooders, M. (Eds.), Group Formation in Economics: Networks, Clubs and Coalitions. Cambridge University Press, Cambridge, pp. 11-57.

Jackson, M.O., van den Nouweland, A., 2005. Strongly stable networks. Games Econ. Behav. 51, 420-444.

Jackson, M.O., Watts, A., 2002. The evolution of social and economic networks. J. Econ. Theory 106, 265-295.

Jackson, M.O., Wolinsky, A., 1996. A strategic model of social and economic networks. J. Econ. Theory 71, 44-74.

Mauleon, A., Vannetelbosch, V., 2004. Farsightedness and cautiousness in coalition formation games with positive spillovers. Theory Dec. 56, 291-324.

Page Jr., F.H., Wooders, M., 2005. Strategic basins of attraction, the path dominance core, and network formation games. Working Paper 05-W09. Department of Economics, Vanderbilt University.

Page Jr., F.H., Wooders, M., Kamat, S., 2005. Networks and farsighted stability. J. Econ. Theory 120, 257-269.

Sengupta, A., Sengupta, K., 1994. Viable proposals. Int. Econ. Rev. 35, 347-359.

Tercieux, O., Vannetelbosch, V., 2006. A characterization of stochastically stable networks. Int. J. Game Theory 34, 351-369.

von Neumann, J., Morgenstern, O., 1944. Theory of Games and Economic Behavior. Princeton University Press, Princeton.

Watts, A., 2002. Non-myopic formation of circle networks. Econ. Letters 74, 277-282.

Xue, L., 1998. Coalitional stability under perfect foresight. Econ. Theory 11, 603-627. 